hep-ep/9508013

DESY 95-156

ISSN $0418-9833$

August 1995

\title{
Comparison of Deep Inelastic Scattering with Photoproduction Interactions at HERA
}

\author{
H1 Collaboration
}

\begin{abstract}
Photon-proton $(\gamma p)$ interactions with $Q^{2}<10^{-2} \mathrm{GeV}^{2}$ and deep-inelastic scattering $\left(\gamma^{*} p\right)$ interactions with photon virtualities $Q^{2}>5 \mathrm{GeV}^{2}$ are studied at the high energy electron-proton collider HERA. The transverse energy flow and relative rates of large rapidity gap events are compared in the two event samples. The observed similarity between $\gamma p$ and $\gamma^{*} p$ interactions can be understood in a picture where the photon develops as a hadronic object. The transverse energy density measured in the central region of the collision, at $\eta^{*}=0$ in the $\gamma^{*} p$ centre of mass frame, is compared with data from hadron-hadron interactions as function of the CMS energy of the collision.
\end{abstract}




\section{H1 Collaboration}

S. Aid ${ }^{14}$, V. Andreev ${ }^{26}$, B. Andrieu ${ }^{29}$, R.-D. Appuhn ${ }^{12}$, M. Arpagaus ${ }^{37}$, A. Babaev ${ }^{25}$, J. Bähr ${ }^{36}$, J. Bán ${ }^{18}$, Y. Ban ${ }^{28}$, P. Baranov ${ }^{26}$, E. Barrelet ${ }^{30}$, R. Barschke ${ }^{12}$, W. Bartel ${ }^{12}$, M. Barth ${ }^{5}$, U. Bassler ${ }^{30}$, H.P. Beck ${ }^{38}$, H.-J. Behrend ${ }^{12}$, A. Belousov ${ }^{26}$, Ch. Berger ${ }^{1}$, G. Bernardi ${ }^{30}$, R. Bernet ${ }^{37}$, G. Bertrand-Coremans ${ }^{5}$, M. Besançon ${ }^{10}$, R. Beyer $^{12}$, P. Biddulph ${ }^{23}$, P. Bispham ${ }^{23}$, J.C. Bizot ${ }^{28}$, V. Blobel ${ }^{14}$, K. Borras ${ }^{9}$, F. Botterweck ${ }^{5}$, V. Boudry ${ }^{29}$, S. Bourov ${ }^{25}$, A. Braemer ${ }^{15}$, F. Brasse ${ }^{12}$, W. Braunschweig ${ }^{1}$, V. Brisson ${ }^{28}$, D. Bruncko ${ }^{18}$, C. Brune ${ }^{16}$, R. Buchholz ${ }^{12}$, L. Büngener ${ }^{14}$, J. Bürger ${ }^{12}$, F.W. Büsser ${ }^{14}$, A. Buniatian ${ }^{12,39}$, S. Burke ${ }^{19}$, M.J. Burton ${ }^{23}$, G. Buschhorn ${ }^{27}$, A.J. Campbell ${ }^{12}$, T. Carli ${ }^{27}$, F. Charles $^{12}$, M. Charlet ${ }^{12}$, D. Clarke $^{6}$, A.B. Clegg ${ }^{19}$, B. Clerbaux ${ }^{5}$, J.G. Contreras ${ }^{9}$, C. Cormack ${ }^{20}$, J.A. Coughlan ${ }^{6}$, A. Courau ${ }^{28}$, Ch. Coutures ${ }^{10}$, G. Cozzika ${ }^{10}$, L. Criegee ${ }^{12}$, D.G. Cussans ${ }^{6}$, J. Cvach ${ }^{31}$, S. Dagoret ${ }^{30}$, J.B. Dainton ${ }^{20}$, W.D. Dau ${ }^{17}$, K. Daum ${ }^{35}$, M. David ${ }^{10}$, C.L. Davis ${ }^{19}$, B. Delcourt ${ }^{28}$, L. Del Buono ${ }^{30}$, A. De Roeck ${ }^{12}$, E.A. De Wolf ${ }^{5}$, P. Dixon ${ }^{19}$, P. Di Nezza ${ }^{33}$, C. Dollfus ${ }^{38}$, J.D. Dowell ${ }^{4}$, H.B. Dreis ${ }^{2}$, A. Droutskoi ${ }^{25}$, J. Duboc ${ }^{30}$, D. Düllmann ${ }^{14}$, O. Dünger ${ }^{14}$, H. Duhm ${ }^{13}$, J. Ebert ${ }^{35}$, T.R. Ebert ${ }^{20}$, G. Eckerlin ${ }^{12}$, V. Efremenko ${ }^{25}$, S. Egli ${ }^{38}$, H. Ehrlichmann ${ }^{36}$, S. Eichenberger ${ }^{38}$, R. Eichler ${ }^{37}$, F. Eisele ${ }^{15}$, E. Eisenhandler ${ }^{21}$, R.J. Ellison ${ }^{23}$, E. Elsen ${ }^{12}$, M. Erdmann ${ }^{15}$, W. Erdmann ${ }^{37}$, E. Evrard ${ }^{5}$, L. Favart ${ }^{5}$, A. Fedotov ${ }^{25}$, D. Feeken ${ }^{14}$, R. Felst ${ }^{12}$, J. Feltesse ${ }^{10}$, J. Ferencei ${ }^{16}$, F. Ferrarotto ${ }^{33}$, K. Flamm ${ }^{12}$, M. Fleischer ${ }^{27}$, M. Flieser ${ }^{27}$, G. Flügge ${ }^{2}$, A. Fomenko ${ }^{26}$, B. Fominykh ${ }^{25}$, M. Forbush ${ }^{8}$, J. Formánek ${ }^{32}$, J.M. Foster ${ }^{23}$, G. Franke ${ }^{12}$, E. Fretwurst ${ }^{13}$, E. Gabathuler ${ }^{20}$, K. Gabathuler ${ }^{34}$,

J. Garvey ${ }^{4}$, J. Gayler ${ }^{12}$, M. Gebauer ${ }^{9}$, A. Gellrich ${ }^{12}$, H. Genzel ${ }^{1}$, R. Gerhards ${ }^{12}$, A. Glazov ${ }^{36}$, U. Goerlach ${ }^{12}$, L. Goerlich ${ }^{7}$, N. Gogitidze ${ }^{26}$, M. Goldberg ${ }^{30}$, D. Goldner ${ }^{9}$, B. Gonzalez-Pineiro ${ }^{30}$, I. Gorelov ${ }^{25}$, P. Goritchev ${ }^{25}$, C. Grab ${ }^{37}$, H. Grässler ${ }^{2}$, R. Grässler ${ }^{2}$, T. Greenshaw ${ }^{20}$, R. Griffiths ${ }^{21}$, G. Grindhammer ${ }^{27}$, A. Gruber ${ }^{27}$, C. Gruber ${ }^{17}$, J. Haack ${ }^{36}$, D. Haidt ${ }^{12}$, L. Hajduk ${ }^{7}$, O. Hamon ${ }^{30}$, M. Hampel $^{1}$, M. Hapke ${ }^{12}$, W.J. Haynes ${ }^{6}$, J. Heatherington ${ }^{21}$, G. Heinzelmann ${ }^{14}$, R.C.W. Henderson ${ }^{19}$, H. Henschel ${ }^{36}$, I. Herynek ${ }^{31}$, M.F. Hess ${ }^{27}$, W. Hildesheim ${ }^{12}$, P. Hill ${ }^{6}$, K.H. Hiller ${ }^{36}$, C.D. Hilton ${ }^{23}$, J. Hladký ${ }^{31}$, K.C. Hoeger ${ }^{23}$, M. Höppner ${ }^{9}$, R. Horisberger ${ }^{34}$, V.L. Hudgson ${ }^{4}$, Ph. Huet ${ }^{5}$, M. Hütte ${ }^{9}$, H. Hufnagel ${ }^{15}$, M. Ibbotson ${ }^{23}$, H. Itterbeck ${ }^{1}$, M.-A. Jabiol ${ }^{10}$, A. Jacholkowska ${ }^{28}$, C. Jacobsson ${ }^{22}$, M. Jaffre ${ }^{28}$, J. Janoth ${ }^{16}$, T. Jansen ${ }^{12}$, L. Jönsson ${ }^{22}$, D.P. Johnson ${ }^{5}$, L. Johnson ${ }^{19}$, H. Jung ${ }^{10}$, P.I.P. Kalmus ${ }^{21}$, D. Kant ${ }^{21}$, R. Kaschowitz ${ }^{2}$, P. Kasselmann ${ }^{13}$, U. Kathage ${ }^{17}$, J. Katzy ${ }^{15}$, H.H. Kaufmann ${ }^{36}$, S. Kazarian ${ }^{12}$, I.R. Kenyon ${ }^{4}$, S. Kermiche ${ }^{24}$, C. Keuker ${ }^{1}$, C. Kiesling ${ }^{27}$, M. Klein ${ }^{36}$, C. Kleinwort ${ }^{14}$, G. Knies ${ }^{12}$, W. Ko ${ }^{8}$, T. Köhler ${ }^{1}$, J.H. Köhne ${ }^{27}$, H. Kolanoski ${ }^{3}$, F. Kole ${ }^{8}$, S.D. Kolya ${ }^{23}$, V. Korbel ${ }^{12}$, M. Korn ${ }^{9}$, P. Kostka ${ }^{36}$, S.K. Kotelnikov ${ }^{26}$,

T. Krämerkämper ${ }^{9}$, M.W. Krasny ${ }^{7,30}$, H. Krehbiel ${ }^{12}$, D. Krücker², U. Krüger ${ }^{12}$, U. Krüner-Marquis ${ }^{12}$, H. Küster ${ }^{2}$, M. Kuhlen ${ }^{27}$, T. Kurča ${ }^{18}$, J. Kurzhöfer ${ }^{9}$, B. Kuznik ${ }^{35}$, D. Lacour $^{30}$, B. Laforge ${ }^{10}$, F. Lamarche ${ }^{29}$, R. Lander ${ }^{8}$, M.P.J. Landon ${ }^{21}$, W. Lange ${ }^{36}$, P. Lanius ${ }^{27}$, J.-F. Laporte ${ }^{10}$, A. Lebedev ${ }^{26}$, F. Lehner ${ }^{12}$, C. Leverenz ${ }^{12}$, S. Levonian ${ }^{26}$, Ch. Ley ${ }^{2}$, G. Lindström ${ }^{13}$, J. Link ${ }^{8}$, F. Linsel ${ }^{12}$, J. Lipinski ${ }^{14}$, B. List ${ }^{12}$, G. Lobo ${ }^{28}$, P. $\operatorname{Loch}^{28}$, H. Lohmander ${ }^{22}$, J.W. Lomas ${ }^{23}$, G.C. Lopez ${ }^{21}$, V. Lubimov ${ }^{25}$, D. Lüke ${ }^{9,12}$, N. Magnussen ${ }^{35}$, E. Malinovski ${ }^{26}$, S. Mani ${ }^{8}$, R. Maraček ${ }^{18}$, P. Marage ${ }^{5}$, J. Marks ${ }^{24}$, R. Marshalli23, J. Martens ${ }^{35}$, G. Martin ${ }^{14}$, R. Martin ${ }^{20}$, H.-U. Martyn ${ }^{1}$, J. Martyniak ${ }^{28}$, 
S. Masson ${ }^{2}$, T. Mavroidis ${ }^{21}$, S.J. Maxfield ${ }^{20}$, S.J. McMahon ${ }^{20}$, A. Mehta ${ }^{6}$, K. Meier ${ }^{16}$, D. Mercer $^{23}$, T. Merz ${ }^{36}$, A. Meyer ${ }^{12}$, C.A. Meyer ${ }^{38}$, H. Meyer ${ }^{35}$, J. Meyer ${ }^{12}$,

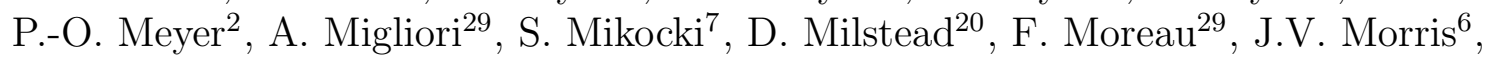
E. Mroczko ${ }^{7}$, G. Müller ${ }^{12}$, K. Müller ${ }^{12}$, P. Murín ${ }^{18}$, V. Nagovizin ${ }^{25}$, R. Nahnhauer ${ }^{36}$, B. Naroska ${ }^{14}$, Th. Naumann ${ }^{36}$, P.R. Newman ${ }^{4}$, D. Newton ${ }^{19}$, D. Neyret ${ }^{30}$, H.K. Nguyen ${ }^{30}$, T.C. Nicholls ${ }^{4}$, F. Niebergall ${ }^{14}$, C. Niebuhr ${ }^{12}$, Ch. Niedzballa ${ }^{1}$,

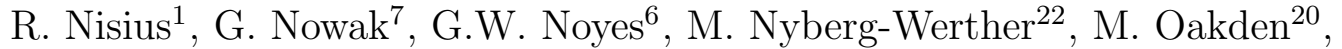
H. Oberlack ${ }^{27}$, U. Obrock ${ }^{9}$, J.E. Olsson ${ }^{12}$, D. Ozerov ${ }^{25}$, P. Palmen ${ }^{2}$, E. Panaro ${ }^{12}$, A. Panitch ${ }^{5}$, C. Pascaud ${ }^{28}$, G.D. Patel ${ }^{20}$, H. Pawletta ${ }^{2}$, E. Peppel ${ }^{36}$, E. Perez ${ }^{10}$, J.P. Phillips ${ }^{20}$, Ch. Pichler ${ }^{13}$, A. Pieuchot ${ }^{24}$, D. Pitzl ${ }^{37}$, G. Pope ${ }^{8}$, S. Prell ${ }^{12}$, R. Prosi ${ }^{12}$, K. Rabbertz ${ }^{1}$, G. Rädel ${ }^{12}$, F. Raupach ${ }^{1}$, P. Reimer ${ }^{31}$, S. Reinshagen ${ }^{12}$, P. Ribarics ${ }^{27}$, H. Rick ${ }^{9}$, V. Riech ${ }^{13}$, J. Riedlberger ${ }^{37}$, S. Riess ${ }^{14}$, M. Rietz ${ }^{2}$, E. Rizvi ${ }^{21}$, S.M. Robertson ${ }^{4}$, P. Robmann ${ }^{38}$, H.E. Roloff ${ }^{36}$, R. Roosen ${ }^{5}$, K. Rosenbauer ${ }^{1}$, A. Rostovtsev ${ }^{25}$, F. Rouse ${ }^{8}$, C. Royon ${ }^{10}$, K. Rüter ${ }^{27}$, S. Rusakov ${ }^{26}$, K. Rybicki ${ }^{7}$, R. Rylko ${ }^{21}$, N. Sahlmann ${ }^{2}$, D.P.C. Sankey ${ }^{6}$, P. Schacht ${ }^{27}$, S. Schiek ${ }^{14}$, S. Schleif ${ }^{16}$, P. Schleper ${ }^{15}$, W. von Schlippe ${ }^{21}$, D. Schmidt ${ }^{35}$, G. Schmidt ${ }^{14}$, A. Schöning ${ }^{12}$, V. Schröder ${ }^{12}$, E. Schuhmann ${ }^{27}$, B. Schwab ${ }^{15}$, G. Sciacca ${ }^{36}$, F. Sefkow ${ }^{12}$, M. Seidel ${ }^{13}$, R. Sell ${ }^{12}$, A. Semenov ${ }^{25}$, V. Shekelyan ${ }^{12}$, I. Sheviakov ${ }^{26}$, L.N. Shtarkov ${ }^{26}$, G. Siegmon ${ }^{17}$, U. Siewert ${ }^{17}$, Y. Sirois ${ }^{29}$, I.O. Skillicorn ${ }^{11}$, P. Smirnov ${ }^{26}$, J.R. Smith ${ }^{8}$, V. Solochenko ${ }^{25}$, Y. Soloviev ${ }^{26}$, J. Spiekermann ${ }^{9}$, S. Spielman ${ }^{29}$, H. Spitzer ${ }^{14}$, R. Starosta ${ }^{1}$, M. Steenbock ${ }^{14}$, P. Steffen ${ }^{12}$, R. Steinberg ${ }^{2}$, B. Stella ${ }^{33}$, K. Stephens ${ }^{23}$, J. Stier ${ }^{12}$, J. Stiewe ${ }^{16}$, U. Stößlein ${ }^{36}$, K. Stolze ${ }^{36}$, J. Strachota ${ }^{31}$, U. Straumann ${ }^{38}$, W. Struczinski ${ }^{2}$, J.P. Sutton ${ }^{4}$, S. Tapprogge ${ }^{16}$, V. Tchernyshov ${ }^{25}$, J. Theissen ${ }^{2}$, C. Thiebaux ${ }^{29}$, G. Thompson ${ }^{21}$, P. Truöl ${ }^{38}$, J. Turnau ${ }^{7}$, J. Tutas ${ }^{15}$, P. Uelkes ${ }^{2}$, A. Usik ${ }^{26}$, S. Valkár ${ }^{32}$, A. Valkárová ${ }^{32}$, C. Vallée ${ }^{24}$, D. Vandenplas ${ }^{29}$, P. Van $\mathrm{Esch}^{5}$, P. Van Mechelen ${ }^{5}$,

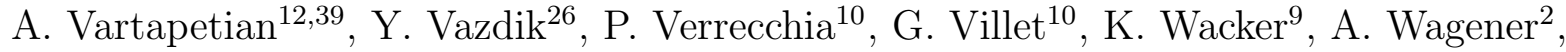
M. Wagener ${ }^{34}$, A. Walther ${ }^{9}$, B. Waugh ${ }^{23}$, G. Weber ${ }^{14}$, M. Weber ${ }^{12}$, D. Wegener ${ }^{9}$, A. Wegner ${ }^{12}$, H.P. Wellisch ${ }^{27}$, L.R. West ${ }^{4}$, S. Willard ${ }^{8}$, M. Winde ${ }^{36}$, G.-G. Winter ${ }^{12}$, C. Wittek ${ }^{14}$, A.E. Wright ${ }^{23}$, E. Wünsch ${ }^{12}$, N. Wulff ${ }^{12}$, T.P. Yiou ${ }^{30}$, J. Žáček ${ }^{32}$, D. Zarbock ${ }^{13}$, Z. Zhang ${ }^{28}$, A. Zhokin ${ }^{25}$, M. Zimmer ${ }^{12}$, W. Zimmermann ${ }^{12}$, F. Zomer ${ }^{28}$, J. Zsembery ${ }^{10}$, K. Zuber ${ }^{16}$, and M. zurNedden ${ }^{38}$

${ }^{1}$ I. Physikalisches Institut der RWTH, Aachen, Germany ${ }^{a}$

${ }^{2}$ III. Physikalisches Institut der RWTH, Aachen, Germany ${ }^{a}$

3 Institut für Physik, Humboldt-Universität, Berlin, Germany ${ }^{a}$

${ }^{4}$ School of Physics and Space Research, University of Birmingham, Birmingham, UK ${ }^{b}$

${ }^{5}$ Inter-University Institute for High Energies ULB-VUB, Brussels; Universitaire Instelling Antwerpen, Wilrijk; Belgium ${ }^{c}$

${ }^{6}$ Rutherford Appleton Laboratory, Chilton, Didcot, $U K^{b}$

7 Institute for Nuclear Physics, Cracow, Poland ${ }^{d}$

8 Physics Department and IIRPA, University of California, Davis, California, USA

9 Institut für Physik, Universität Dortmund, Dortmund, Germany ${ }^{a}$

10 CEA, DSM/DAPNIA, CE-Saclay, Gif-sur-Yvette, France

11 Department of Physics and Astronomy, University of Glasgow, Glasgow, UK ${ }^{b}$

12 DESY, Hamburg, Germany ${ }^{a}$ 
13 I. Institut für Experimentalphysik, Universität Hamburg, Hamburg, Germany ${ }^{a}$

14 II. Institut für Experimentalphysik, Universität Hamburg, Hamburg, Germany ${ }^{a}$

15 Physikalisches Institut, Universität Heidelberg, Heidelberg, Germany ${ }^{a}$

16 Institut für Hochenergiephysik, Universität Heidelberg, Heidelberg, Germany ${ }^{a}$

17 Institut für Reine und Angewandte Kernphysik, Universität Kiel, Kiel, Germany ${ }^{a}$

18 Institute of Experimental Physics, Slovak Academy of Sciences, Košice, Slovak

Republic ${ }^{f}$

19 School of Physics and Chemistry, University of Lancaster, Lancaster, $U K^{b}$

20 Department of Physics, University of Liverpool, Liverpool, UK ${ }^{b}$

21 Queen Mary and Westfield College, London, UK

22 Physics Department, University of Lund, Lund, Sweden ${ }^{g}$

23 Physics Department, University of Manchester, Manchester, UK ${ }^{b}$

${ }^{24}$ CPPM, Université d'Aix-Marseille II, IN2P3-CNRS, Marseille, France

25 Institute for Theoretical and Experimental Physics, Moscow, Russia

${ }^{26}$ Lebedev Physical Institute, Moscow, Russia ${ }^{f}$

27 Max-Planck-Institut für Physik, München, Germany ${ }^{a}$

${ }^{28}$ LAL, Université de Paris-Sud, IN2P3-CNRS, Orsay, France

${ }^{29}$ LPNHE, Ecole Polytechnique, IN2P3-CNRS, Palaiseau, France

${ }^{30}$ LPNHE, Universités Paris VI and VII, IN2P3-CNRS, Paris, France

31 Institute of Physics, Czech Academy of Sciences, Praha, Czech Republic ${ }^{f, h}$

${ }^{32}$ Nuclear Center, Charles University, Praha, Czech Republic ${ }^{f, h}$

33 INFN Roma and Dipartimento di Fisica, Universita "La Sapienza", Roma, Italy

34 Paul Scherrer Institut, Villigen, Switzerland

35 Fachbereich Physik, Bergische Universität Gesamthochschule Wuppertal, Wuppertal, Germany ${ }^{a}$

${ }^{36}$ DESY, Institut für Hochenergiephysik, Zeuthen, Germany ${ }^{a}$

37 Institut für Teilchenphysik, ETH, Zürich, Switzerland

38 Physik-Institut der Universität Zürich, Zürich, Switzerland

39 Visitor from Yerevan Phys.Inst., Armenia

${ }^{a}$ Supported by the Bundesministerium für Forschung und Technologie, FRG under contract numbers 6AC17P, 6AC47P, 6DO57I, 6HH17P, 6HH27I, 6HD17I, 6HD27I, 6KI17P, 6MP17I, and 6WT87P

${ }^{b}$ Supported by the UK Particle Physics and Astronomy Research Council, and formerly by the UK Science and Engineering Research Council

c Supported by FNRS-NFWO, IISN-IIKW

$d$ Supported by the Polish State Committee for Scientific Research, grant Nos. SPUB/P3/202/94 and 2 PO3B 237 08, and Stiftung fuer Deutsch-Polnische

Zusammenarbeit, project no.506/92

e Supported in part by USDOE grant DE F603 91 ER40674

$f$ Supported by the Deutsche Forschungsgemeinschaft

$g$ Supported by the Swedish Natural Science Research Council

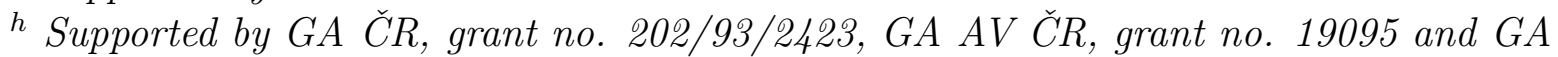
UK, grant no. 342

i Supported by the Swiss National Science Foundation 


\section{Introduction}

Photon-proton processes are traditionally classified according to the virtuality $\left(Q^{2}\right)$ of the photon. For quasi-real photoproduction interactions $Q^{2}$ is close to zero, and correspondingly the photon is nearly on mass shell. Interactions which involve a $Q^{2}$ larger than a few $\mathrm{GeV}^{2}$ are usually termed deep-inelastic scattering processes (DIS). This distinction results mainly from the different theoretical descriptions adopted for these processes. Photoproduction has turned out to be very similar to hadron-hadron collisions and is described in a VMD-like (Vector Meson Dominance) picture [1], where the photon is assumed to fluctuate into a vector meson before interacting with the proton. Additional diagrams such as the direct coupling of the photon to quarks in the proton and a pointlike contribution where the photon splits into a $q \bar{q}$ pair are needed to accommodate the large tail observed in the transverse momentum distribution of produced particles [2], but the majority of photoproduction collisions show features of soft low- $p_{T}$ processes as seen in hadronic collisions. For DIS on the other hand, the observation of scaling of the structure function in early experiments suggested that this interaction could, in the infinite momentum frame of the proton, be interpreted as a hard scattering process in which a point-like virtual photon "probes" the structure of the hadronic target. The subsequently measured scaling violations are well described by perturbative QCD.

However, recent DIS measurements at the high energy ep collider HERA and high precision measurements from fixed target leptoproduction experiments have shown that the data in the low Bjorken- $x$ region reveal properties from soft interactions as well. Here $x=Q^{2} / 2 P \cdot q$ where $P$ and $q$ are the four momenta of the incoming proton and photon, respectively. Elastic and other diffractive hadronic final states have been produced [3, (4] and shadowing on nuclear targets has been observed [5], phenomena which are well known from hadron-hadron and real photon collisions [6]. These observations suggest that the different treatment of low and high $Q^{2}$ interactions is somewhat artificial, and it is therefore worthwhile testing a prescription which provides a natural transition between these two classes.

A qualitative approach for a smooth transition of the high energy $\gamma^{*} p$ H interactions over a large range of $Q^{2}$ results from viewing the photon-proton collision in the proton rest frame and the hypothesis that the photon can develop as a hadronic object before interacting with the nuclear target. The time in which a real photon can fluctuate into e.g. a $\rho$ meson is given by the Heisenberg uncertainty principle and amounts to $\tau \approx 2 \nu / M_{\rho}^{2}$ where $\nu$ is the photon energy in the proton rest frame and $M_{\rho}$ the mass of the $\rho$ meson. The lifetime of a high energy real photon to fluctuate into a hadronic state is much longer than the time of the strong interaction itself, and therefore this picture is generally applied to describe photoproduction interactions. The same argument can be used for DIS interactions at low $x$. In the proton rest frame the time in which the virtual photon can fluctuate and stay in a hadronic state, e.g. a quark-antiquark pair, is given by $\tau \approx 1 /\left(x M_{p}\right)$ [7, 8], where $M_{p}$ is the mass of the nucleon target. Thus, for small $x$, the virtual photon can convert into a quark-antiquark pair and cover a distance which is long compared to the interaction length. For an $x$ in the range of $10^{-2}-10^{-4}$ this distance is in the range of 10 to $1000 \mathrm{fm}$, much larger than the typical radius of the target. In

\footnotetext{
${ }^{1}$ In this paper the generic symbol $\gamma^{*}$ is used to denote a colliding photon irrespective of the virtuality.
} 
the HERA kinematic range such $x$ values can be reached for $Q^{2} \sim 10 \mathrm{GeV}^{2}$. Therefore, at low $x$, a virtual photon can stay in a hadronic state for a long time and interact with the target strongly, leading to a final state similar to the one in a hadron-hadron collision. This picture of DIS in the frame where the incoming photon fluctuates into a hadronic system before interacting with the proton has already been advocated in various papers [9, 10, 11]. Note that this is not in contradiction with the traditional treatment of DIS as a point-like process, where a virtual photon "probes" the hadronic structure of the nuclear target. In fact both pictures are taken to be complementary, as discussed in [8].

The HERA collider provides a unique opportunity to study the final state of both photoproduction and DIS interactions at high energy and small Bjorken- $x$ : $26.7 \mathrm{GeV}$ electrons collide with $820 \mathrm{GeV}$ protons, yielding a centre of mass energy $\sqrt{s}$ of $296 \mathrm{GeV}$. This allows for a study of DIS interactions for $x$ values down to $\sim 10^{-4}$ [12], and photoproduction collisions at a centre of mass energy of $\sim 200 \mathrm{GeV}$ [2]. The data recorded with the $\mathrm{H} 1$ experiment are used to study the transverse energy behaviour in photoproduction and DIS interactions, and the analogy with hadronic collisions is checked. Within this analogy $\gamma^{*} p$ events can be sub-divided in rapidity-space into three regions, which differ in the mechanism by which the hadronic final state is produced [13]: the proton fragmentation region, the photon fragmentation region and a hadron plateau spanning the rapidity interval between the two. The height of the hadronic plateau depends logarithmically on the centre of mass energy of the $\gamma^{*} p$ collision. The region of the hadronic plateau is expected to be independent of the nature of the two incoming "hadrons", as was verified in hadron-hadron collisions [14. Particle production in the proton fragmentation region is also expected to be very similar compared to hadron-proton collisions, but the limited experimental acceptance in this region does not allow this to be verified. The "hadronic" nature of the photon is assumed to change with increasing $Q^{2}$, thus the photon fragmentation region is expected to change with $Q^{2}$.

Also studied is the fraction of diffractive events in DIS and photoproduction. In hadronic collisions approximate factorization of the cross section for high mass diffractive dissociation has been observed, in accordance with Regge theory predictions [15]: the ratio of the hadron diffractive dissociation to the total cross section is found to be approximately independent of the type of dissociating hadron and is thus the same for pions, kaons, protons and real photons [16]. Applying this factorization property to real and virtual photons leads to the expectation that the ratio of the photon diffractive dissociation cross section to the total cross section is independent of the $Q^{2}$ of the photon, in the high dissociative mass region.

\section{Detector Description}

A detailed description of the H1 apparatus can be found elsewhere [17]. In the following the components of the detector relevant for this analysis are briefly described.

The hadronic energy flow and the scattered electrons in DIS processes are measured with a liquid argon (LAr) calorimeter and a backward electromagnetic lead-scintillator calorimeter (BEMC). The LAr calorimeter [18] covers the polar angular range $4^{\circ}<\theta<$ $153^{\circ}$ with full azimuthal coverage, where $\theta$ is defined with respect to the proton beam 
direction ( $+z$ axis). It consists of an electromagnetic section with lead absorbers and a hadronic section with steel absorbers. Both sections are highly segmented in the transverse and longitudinal direction with about 44000 cells in total. The total depth of both sections varies between 4.5 and 8 interaction lengths. Test beam measurements of the LAr calorimeter modules show an energy resolution of $\sigma_{E} / E \approx 0.12 / \sqrt{E[\mathrm{GeV}]} \oplus 0.01$ for electrons [19] and $\sigma_{E} / E \approx 0.50 / \sqrt{E[\mathrm{GeV}]} \oplus 0.02$ for charged pions [20]. The uncertainty in the absolute energy scale for electrons is $3 \%$. The absolute scale of the hadronic energy is presently known to $5 \%$, as determined from studies of the transverse momentum balance in DIS events.

The BEMC (depth of 22.5 radiation lengths or 1 interaction length) covers the backward region of the detector, $151^{\circ}<\theta<177^{\circ}$. A major task of the BEMC is to trigger on and measure scattered electrons in DIS processes with $Q^{2}$ values ranging from 5 to 100 $\mathrm{GeV}^{2}$. The BEMC energy scale for electrons is known to an accuracy of $1.7 \%$ [21]. Its resolution is given by $\sigma_{E} / E=0.10 / \sqrt{E[\mathrm{GeV}]} \oplus 0.42 / E[\mathrm{GeV}] \oplus 0.03$ 22.

The calorimeters are surrounded by a superconducting solenoid providing a uniform magnetic field of $1.15 \mathrm{~T}$ parallel to the beam axis in the tracking region. Charged particle tracks are measured in a central drift chamber and the forward tracking system, covering the polar angular range $7^{\circ}<\theta<165^{\circ}$. The central chamber is interleaved with an inner and an outer double layer of multi-wire proportional chambers (MWPC), which were used for the trigger to select events with charged tracks pointing to the interaction region. A backward proportional chamber (BPC) in front of the BEMC with an angular acceptance of $155.5^{\circ}<\theta<174.5^{\circ}$ serves to support electron identification and to precisely measure electron direction. Using information from the $\mathrm{BPC}$, the $\mathrm{BEMC}$ and the reconstructed event vertex the polar angle of the scattered electron is known to better than 2 mrad.

A small angle detector (electron tagger), which is part of the luminosity system, is

a $\mathrm{TlCl} / \mathrm{TlBr}$ crystal calorimeter with an energy resolution $\sigma_{E} / E=0.1 / \sqrt{E[\mathrm{GeV}]}$. It is located at $z=-33 \mathrm{~m}$ and accepts electrons from photoproduction processes with an energy fraction between 0.2 and 0.8 with respect to the beam energy and scattering angles $\theta^{\prime}<5 \operatorname{mrad}\left(\theta^{\prime}=\pi-\theta\right)$.

\section{Event Selection and Correction for Detector Ef- fects}

The data used in this analysis were collected in 1993 and correspond to an integrated luminosity of about $0.3 \mathrm{pb}^{-1}$. The kinematic variables $Q^{2}, x$ and $y$ of the ep collision are determined from the scattered electron: $Q^{2}=4 E_{e} E_{e}^{\prime} \cos ^{2}\left(\theta_{e} / 2\right)$ and $y=1-\left(E_{e}^{\prime} / E_{e}\right)$. $\sin ^{2}\left(\theta_{e} / 2\right)$, where $E_{e}$ is the energy of the incident electron and $E_{e}^{\prime}$ and $\theta_{e}$ are the energy and the polar angle of the scattered electron respectively. The variable $y$ represents the fraction of the energy of the electron transferred to the proton, in the proton rest frame. The scaling variable $x$ is then derived via $x=Q^{2} /(y s)$, and the total hadronic invariant mass is given by $W^{2}=s y-Q^{2}$.

The data are classified into three event sub-samples depending on the $Q^{2}$ range. A different detector component of $\mathrm{H} 1$ is used to detect the scattered electron in each of these 


\begin{tabular}{|c|c|c|}
\hline $\begin{array}{c}\text { photoproduction } \\
\text { sample }\end{array}$ & $\begin{array}{c}\text { low } Q^{2} \text { DIS } \\
\text { sample }\end{array}$ & $\begin{array}{c}\text { high } Q^{2} \text { DIS } \\
\text { sample }\end{array}$ \\
\hline$Q^{2}<10^{-2} \mathrm{GeV}^{2}$ & $5<Q^{2}<100 \mathrm{GeV}^{2}$ & $Q^{2}>100 \mathrm{GeV}^{2}$ \\
\hline$\theta_{e}-\pi<5 \mathrm{mrad}$ & $157^{\circ}<\theta_{e}<173^{\circ}$ & $10^{\circ}<\theta_{e}<148^{\circ}$ \\
\hline $0.3<y<0.5$ & $E_{e}^{\prime}>12 \mathrm{GeV}$ & $0.05<y<0.7$ \\
& $y>0.05$ & \\
\hline
\end{tabular}

Table 1: Accepted kinematic regions for the three sub-samples used in this analysis.

ranges. In the studies below these subsamples get further sub-divided into samples with different $y$ or $Q^{2}$ values.

- The photoproduction sub-sample $\left(Q^{2}<10^{-2} \mathrm{GeV}^{2}\right)$ consists of events where the scattered electron is detected in the small angle electron detector. To avoid regions of low acceptance and to facilitate the data correction procedure, the kinematic range was further restricted to $0.3<y<0.5$. The photoproduction events are triggered by a coincidence of an energy deposit in the small angle electron detector and at least one track pointing to the vertex region. The track condition is derived from the cylindrical MWPC and requires a $p_{T} \gtrsim 200 \mathrm{MeV} / \mathrm{c}$.

- The low $Q^{2}$ DIS sub-sample $\left(5<Q^{2}<100 \mathrm{GeV}^{2}\right)$ consists of events where the scattered electron is detected in the BEMC. The events are triggered by requiring a cluster of more than $4 \mathrm{GeV}$ in the BEMC. The most energetic BEMC cluster is taken to be the scattered electron.

- For the high $Q^{2}$ DIS sub-sample $\left(Q^{2}>100 \mathrm{GeV}^{2}\right)$ the scattered electron is detected in the LAr calorimeter. The events are triggered by requiring a cluster of more than $5 \mathrm{GeV}$ in the electromagnetic part of the LAr calorimeter, and no associated hadronic energy. The electromagnetic cluster in the LAr calorimeter with the highest transverse energy is considered to be the scattered electron.

Further details on the scattered electron identification procedure in the BEMC and LAr calorimeters and in the small angle electron detector can be found in [12, 23, 24], respectively.

The selected kinematic regions for each sub-sample, as given in Table 1, are chosen to ensure a large acceptance, high trigger efficiency and, for the DIS sub-samples, a small photoproduction background (less than 3\%). For all three sub-samples the $z$ position of the event vertex reconstructed from charged tracks was required to be within $\pm 30 \mathrm{~cm}$ of the nominal interaction point. A minimum $y$ cut, $y>0.05$ was imposed for the DIS sub-samples. Events suffering from QED radiation or from a badly reconstructed electron are strongly reduced by requiring that they also fulfill this cut if $y$ is calculated from the measured hadrons.

The final event samples contain 82850 photoproduction events, 15324 low $Q^{2}$ DIS events with $5<Q^{2}<100 \mathrm{GeV}^{2}\left(10^{-4}<x<10^{-2}\right)$, 692 high $Q^{2}$ DIS events with $Q^{2}>100 \mathrm{GeV}^{2}\left(10^{-3}<x<10^{-1}\right)$.

The data are corrected for detector effects using samples of Monte Carlo generated events which were fully simulated in the H1 detector. The PHOJET [25] generator for 
photoproduction and the CDM [26] (Colour Dipole Model) generator for DIS processes were used. The program PHOJET generates $\gamma p$ interactions, treating the photon as a hadron-like object. The model used to simulate the hadronic final states is similar to that used in the Monte Carlo program DTUJET [27] which simulates particle production in $p p$ and $\bar{p} p$ collisions up to very high energies. The CDM Monte Carlo program generates DIS events, and uses the colour dipole model for QCD radiation in the hadronic final state. Here the final state is assumed to be a chain of independently radiating dipoles formed by emitted gluons [28]. Since all radiation is assumed to originate from the dipole formed by the struck quark and the remnant, photon-gluon fusion events have to be added and are taken from the QCD matrix elements [29]. Version 4.03 of the ARIADNE program was used for the CDM studies in this paper.

To estimate systematic uncertainties of the correction procedure for DIS events another model for the hadronic final state was used as well: the MEPS (Matrix Elements plus Parton Showers) model [29]. This model incorporates QCD matrix elements up to first order, with additional soft emissions generated by adding leading log parton showers. Divergences of the matrix element are avoided by imposing a lower limit on the partonparton invariant masses, which was parametrized as a function of $W$ such that it is always $2 \mathrm{GeV}$ above the region in phase space where the order $\alpha_{s}$ contributions would exceed the total cross section. More details on this implementation are given in [30].

\section{Results}

For comparisons of event properties at different $Q^{2}$ values, the $\gamma^{*} p$ centre of mass system (CMS) is chosen as frame of reference. The orientation of the CMS is such that the direction of the proton defines the positive $z^{\prime}$ axis. Transverse quantities are defined with respect to the proton direction in this frame.

The flow of transverse energy, $E_{T}$, as a function of pseudorapidity $\eta^{*}=-\ln \left(\tan \frac{\theta^{*}}{2}\right)$ in the CMS is shown in Fig. 1. Here $\theta^{*}$ is the polar angle of a particle in the CMS frame with respect to the proton direction. The transverse energy is calculated from the energy deposits in the calorimeter cells. For this study the DIS data are restricted to the same kinematic region $0.3<y<0.5$ as the photoproduction events. The $y$ range corresponds to a mean $\gamma^{*} p$ collision energy of $\sqrt{s_{\gamma p}}=W \approx 185 \mathrm{GeV}$. The corrections which have been applied to correct for detector effects never exceed $30 \%$.

Only statistical errors are shown in Fig. 1. For the comparison of the $E_{T}$ spectra at the four different $Q^{2}$ values it is important to identify the relative systematic uncertainty, so the systematic errors on each " $Q^{2}$ point" at a given $\eta^{*}$ value are considered in two parts. Firstly there is a point-to-point systematic error, which has a different effect on each of the four measurements, accounting for kinematically dependent systematic effects. Secondly there is an overall scale error which affects all four points at a given $\eta^{*}$ value in the same way. The main source of the point-to-point systematic error is the model dependence of the correction for detector effects. This dependence was investigated with the CDM and MEPS models and leads to a point-to-point error of 6\%?. Additionally,

\footnotetext{
${ }^{2}$ In the central region the correction for photoproduction events determined with PHOJET is close to the one for DIS events determined with MEPS at $<Q^{2}>\sim 11 \mathrm{GeV}^{2}$
} 


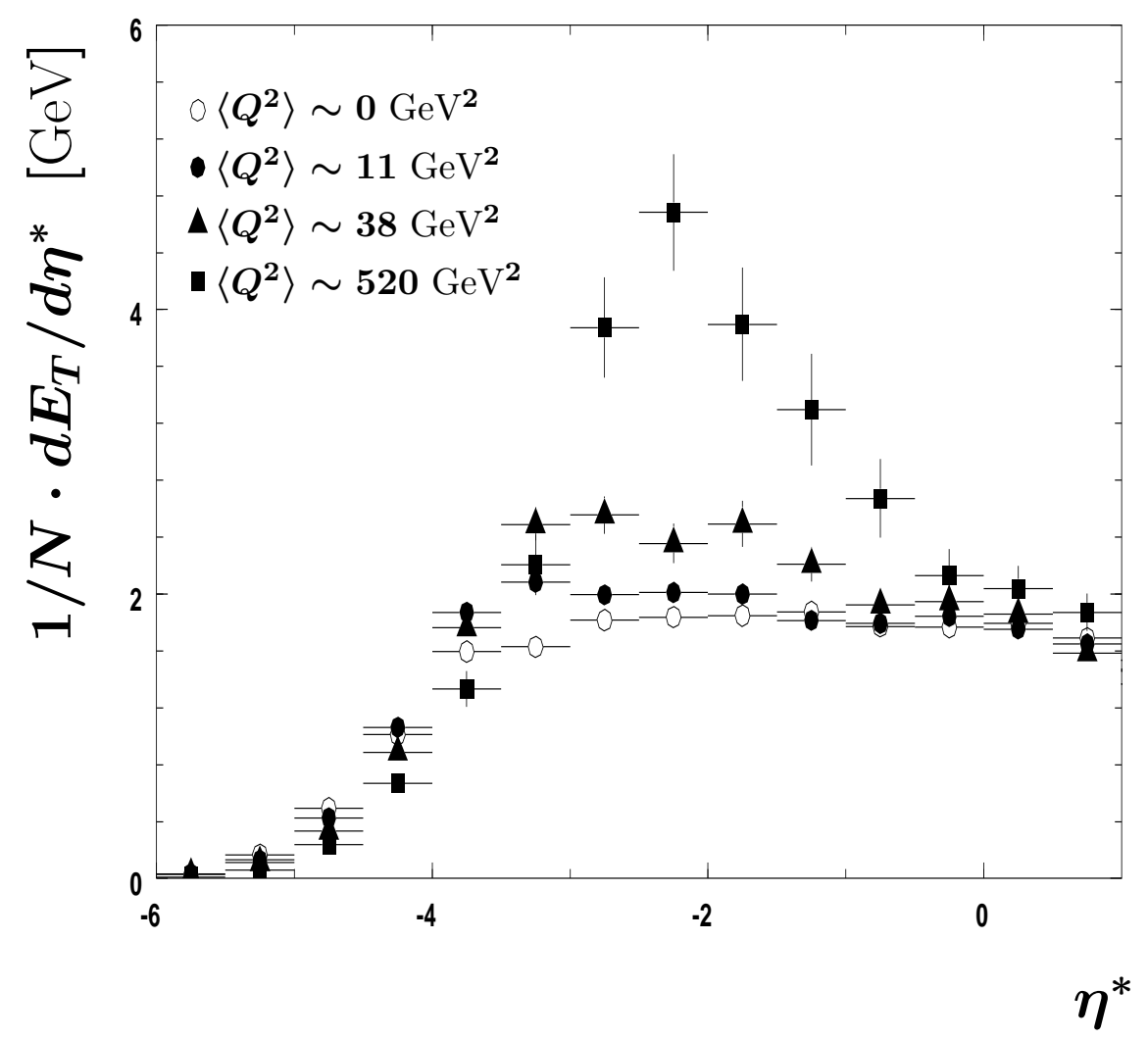

Figure 1: The flow of transverse energy $E_{T}$ in the hadronic CMS as a function of pseudorapidity $\eta^{*}$ normalized to the number of events $N$. Photoproduction data (open circles) are compared with DIS data (full symbols: circles $-\left\langle Q^{2}\right\rangle \approx 11 \mathrm{GeV}^{2}$, triangles $-\left\langle Q^{2}\right\rangle \approx 38 \mathrm{GeV}^{2}$, squares $\left.\left\langle Q^{2}\right\rangle \approx 520 \mathrm{GeV}^{2}\right)$ in the same $y$ range $0.3<y<0.5(W \approx 185 \mathrm{GeV})$.

the model dependence contributes $6 \%$ to the overall scale error at each $\eta^{*}$ value. Further contributions to the overall scale error arise from the LAr and BEMC calorimeter calibration ( $5 \%$ and $20 \%$ respectively), and from details of the analysis method, such as the calorimeter noise treatment, the clustering scheme for the calorimeter cells, and the accuracy of the simulation of the calorimeter response, affecting the results by $5 \%$ in the region close to the proton direction $\left(\eta^{*}>-1\right)$. All these contributions give an overall scale error varying from $8 \%$ in the photon fragmentation region $\left(-3.5<\eta^{*}<-1\right)$ to $9 \%$ in the central region $\left(\eta^{*}>-1\right)$, and up to $20 \%$ in the region $\eta^{*}<-3.5$, which does not affect the results of the present analysis. This discussion on the systematic errors is also valid for data shown below.

Fig. [1 demonstrates that the transverse energy flow exhibits a strong increase in the photon fragmentation region $\left(\eta^{*}<-1\right)$, from $Q^{2}=0$ (photoproduction) to $Q^{2} \approx$ $500 \mathrm{GeV}^{2}$ (DIS), while in the central region the level of $E_{T}$ remains almost the same. This can be taken as evidence that the influence of the $Q^{2}$ of the photon on the $E_{T}$ flow diffuses away quite quickly towards the central region. Such behaviour is expected if the central region corresponds to the hadronic plateau, discussed in the introduction. In order to quantify the change of $E_{T}$ with $Q^{2}$, the value of $E_{T}$ per unit of pseudorapidity ( $E_{T}$ 


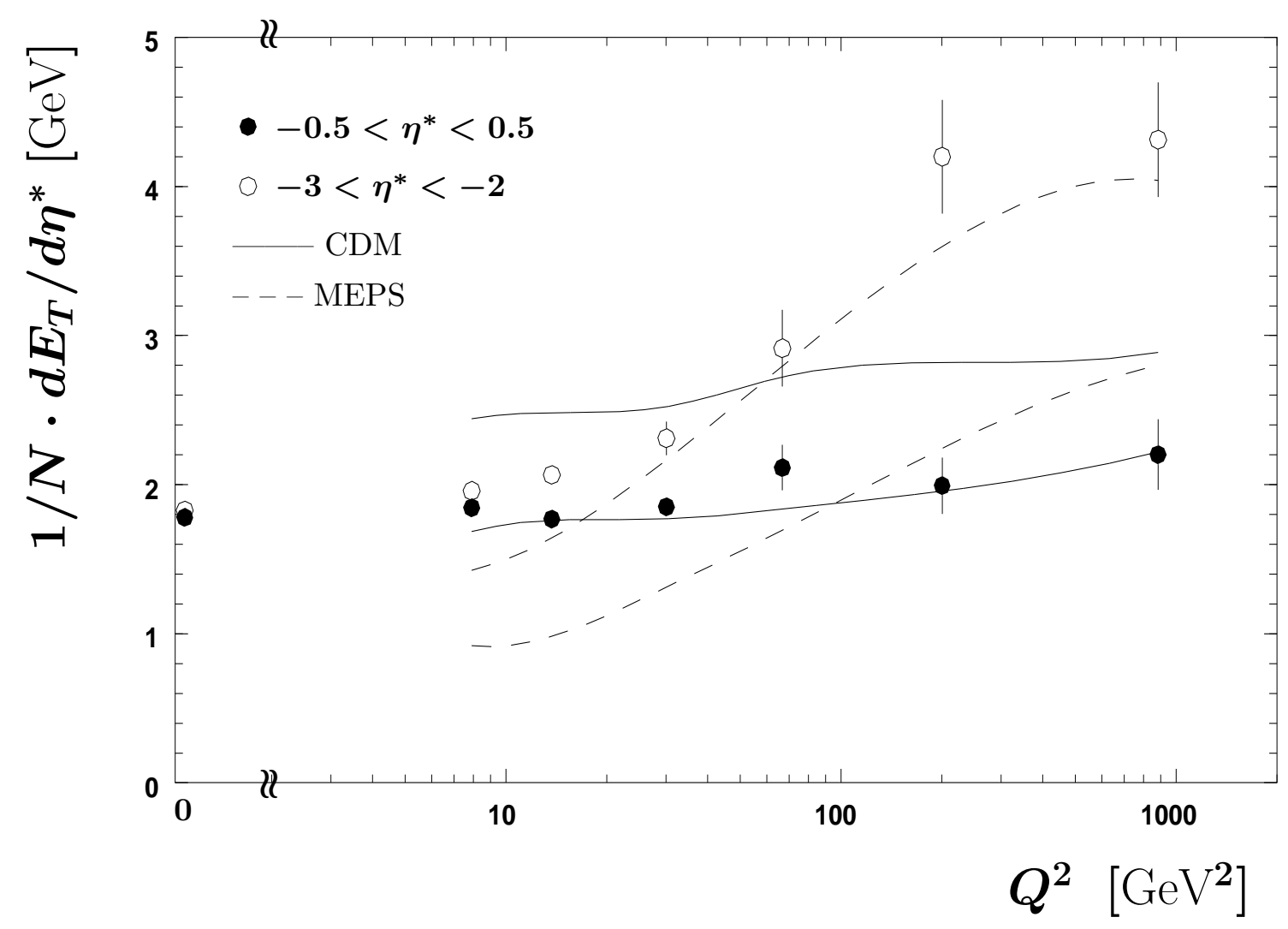

Figure 2: The transverse energy per unit of pseudorapidity in the CMS central region $(-0.5<$ $\eta^{*}<0.5$, full circles) and in the photon fragmentation region $\left(-3<\eta^{*}<-2\right.$, open circles) as a function of $Q^{2}$ for $0.3<y<0.5$. For comparison, the CDM (full line) and MEPS (dashed line) models are shown. The lower curves correspond to the CMS central region and the upper ones to the photon fragmentation region.

density) for an $\eta^{*}$ slice in the central pseudorapidity region $\left(-0.5<\eta^{*}<0.5\right)$ and an $\eta^{*}$ slice in the photon fragmentation region $\left(-3<\eta^{*}<-2\right)$ is shown in Fig. 2. The data clearly demonstrate that the transverse energy in the central region is essentially independent of the $Q^{2}$ of the photon, but increases significantly in the photon fragmentation region. However, in the photon fragmentation region the difference in the $E_{T}$ density for DIS and photoproduction data is significant only for large $Q^{2}$ values, $Q^{2}>20 \mathrm{GeV}^{2}$. The level of transverse energy in the photon fragmentation region for interactions with $Q^{2}<10 \mathrm{GeV}^{2}$ is quite similar, roughly independent of the transverse size, $Q^{2}$, of the photon. Since the former can be described by perturbative calculations, this observation hints that the result of some predicted features of the final state may be transportable to the hitherto assumed non-perturbative "VDM region" at $Q^{2}=0$.

In Fig. 2 the results are compared with predictions from the CDM and MEPS models, as described in the previous section. The $E_{T}$ density predicted by the CDM model is found to be independent of $Q^{2}$, which is in accordance with the data in the central region, but does not reproduce the data in the photon fragmentation region. In this version of the CDM model the scale for $\mathrm{QCD}$ radiation is given by the $p_{T}^{2}$ of the radiated 


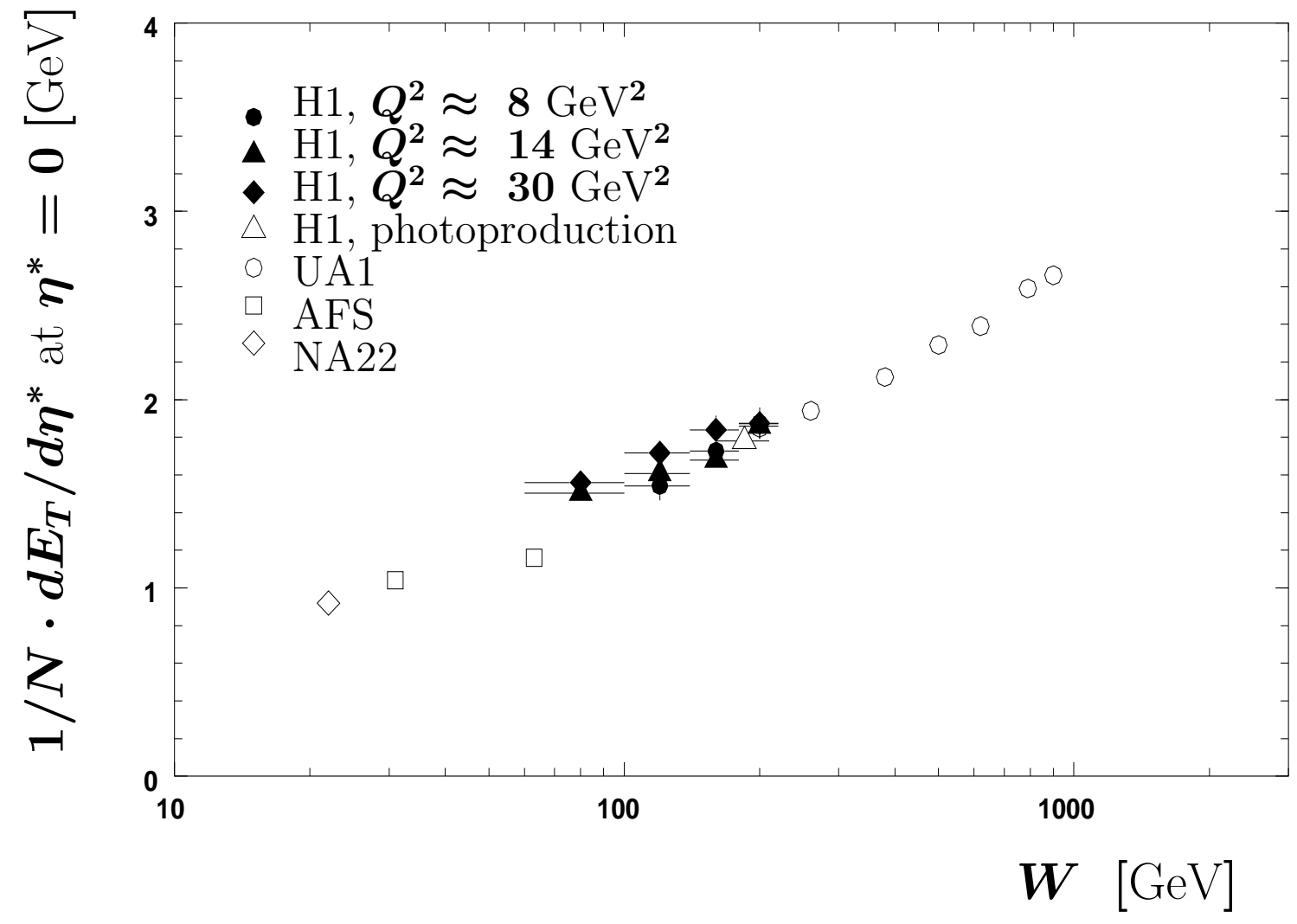

Figure 3: The transverse energy per unit of pseudorapidity in the CMS central region as a function of the hadronic CMS energy. The DIS data are compared with photoproduction data and with data from hadron-hadron collisions ( $p \bar{p}$ for UA1; $p p$ for NA22 and AFS). Systematic point-to-point errors of $6 \%$ and an overall scale error of $9 \%$ for the $\mathrm{H} 1$ data are not shown, neither are the global scale uncertainties for the other experiments.

gluons, which is limited by $W^{4 / 3}$. Since data corresponding to the same $W^{2}$ region are selected, the predictions for the $E_{T}$ density are similar for the different $Q^{2}$ data samples. A new version of the CDM model (version 4.06) [31] partially corrects for this defect in the photon fragmentation region, but still does not yield a good description of the data. The MEPS model on the other hand describes the behaviour of the transverse energy flow in the photon fragmentation region but fails in the central region. Hence, in this model, the influence of the photon virtuality extends too far in rapidity away from the photon- $q \bar{q}$ vertex. The comparison of absolute values of the $E_{T}$ density between the data and models should be viewed with some caution since diffractive events are not included in these models. These events, which amount to $\approx 6 \%$ of all events, give rise to a rapidity gap [3], i.e. no energy deposition in the central region. The exclusion of diffractive events however does not change the shape of the distribution of the $E_{T}$ density versus $Q^{2}$. Comparisons of the energy flows for models and data with rapidity gap events removed can be found in [32].

Within the hadronic picture, the observation of a rise of the $E_{T}$ density with $Q^{2}$ in the photon fragmentation region implies that, on arrival at the target, the virtual photon has 

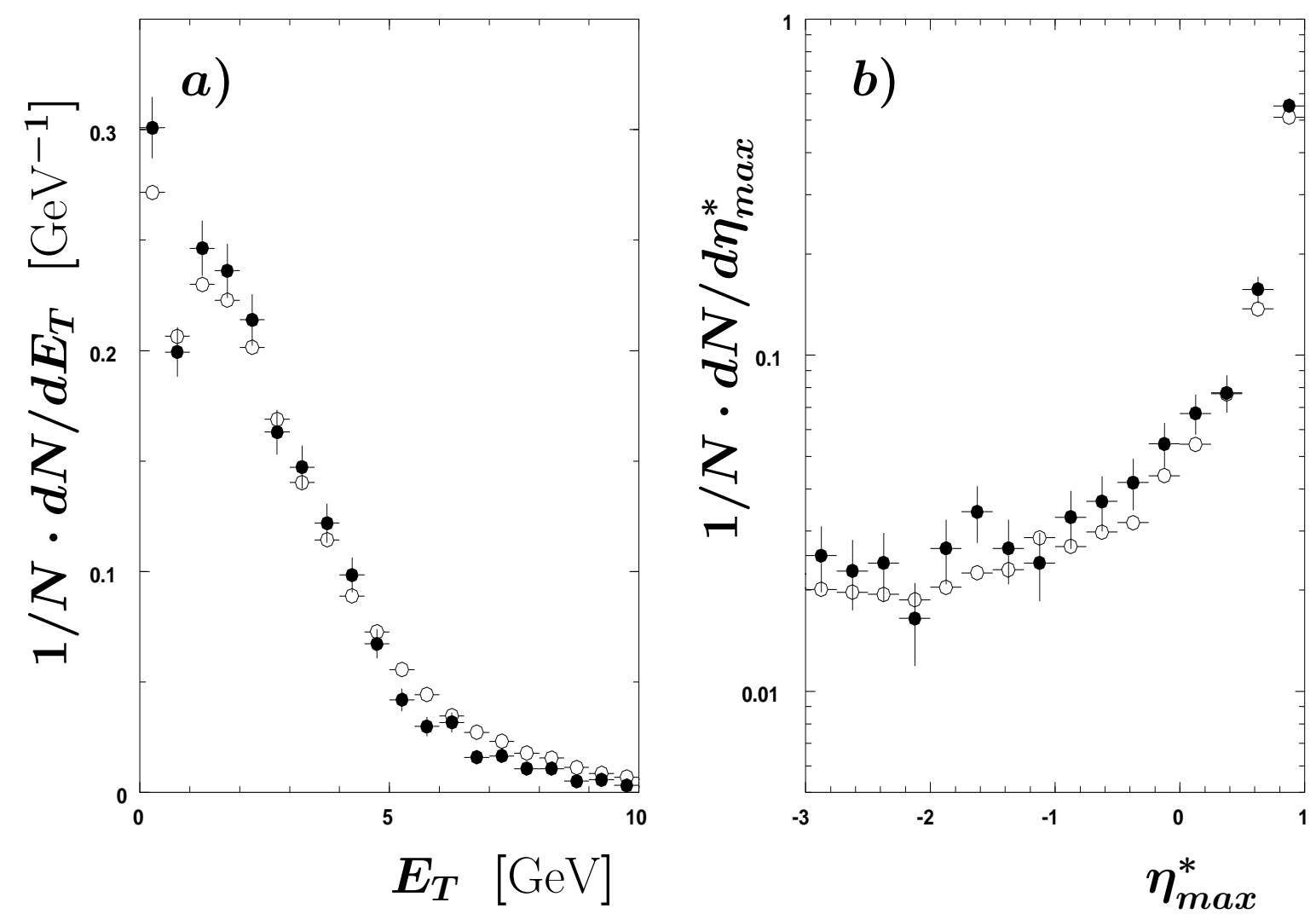

Figure 4: $(a)$ distribution of the uncorrected transverse energy per unit of pseudorapidity in the central region $\left(-0.5<\eta^{*}<0.5\right),(b) \eta_{\max }^{*}$ distribution normalized to the total number of events. Open circles - photoproduction data, full circles - DIS data with $10<Q^{2}<100 \mathrm{GeV}^{2}$ and $0.3<y<0.5$.

a hadronic structure (parton configuration) which depends on $Q^{2}$. With increasing $Q^{2}$ the transverse momentum of the constituent partons in this hadronic structure increases. A formal explanation of this effect based on QCD calculations is given in [33.

The central region however shows a more universal behaviour, independent of the partonic nature of the colliding particles. It is therefore interesting to compare our measurement with high energy hadron-hadron collisions. In Fig. 3 the transverse energy density, $d E_{T} / d \eta^{*}$, at $\eta^{*}=0$ is shown for DIS, photoproduction, $p p$ and $\bar{p} p$ interactions as a function of $W(=\sqrt{s}$ for hadron-hadron collisions). The results for DIS include only the data with $Q^{2}<50 \mathrm{GeV}^{2}$, where enough statistics is available to cover a large $W$ (or equivalently $y$ ) range, as given in Table 1 . The $W$ dependence of $d E_{T} / d \eta^{*}$ observed in DIS processes at low $Q^{2}$ (and low $x$ ) agrees with the $W$ interpolation between measurements from $p \bar{p}$ [34] and $p p$ [34, 35] collisions. Also the photoproduction data show the same level of $E_{T}$ for the $W$ value of this data sample. This observation is consistent with the ansatz of the analogy of $\gamma^{*} p$ interactions with real photon-hadron and hadron-hadron collisions.

In Fig. Wa the distribution of (uncorrected) transverse energy summed over a unit of pseudorapidity in the central region $\left(-0.5<\eta^{*}<0.5\right)$ is shown for photoproduction and for DIS data $\left(10<Q^{2}<100 \mathrm{GeV}^{2}, 0.3<y<0.5\right)$. The comparison shows that in the 
central region not only the mean $E_{T}$, but also the energy spectra themselves, are quite similar. This apparently holds down to very low $E_{T}$ values, where diffractive dissociation processes are expected to dominate (see peak at $E_{T}=0$ ). Since for $\eta^{*}=0$ essentially the same detector regions are used in DIS and photoproduction interactions the agreement is not influenced by detector effects. Following this observation, it is interesting to compare the fraction of diffractive events in photoproduction and DIS.

An effective way to detect diffractive processes at HERA is via the $\eta_{\max }^{*}$ variable, which is the maximum pseudorapidity in the $\gamma^{*} p$ CMS frame, of a reconstructed track or calorimetric cluster with an energy larger than $400 \mathrm{MeV}$ observed in the detector. In diffractive events $\eta_{\max }^{*}$ indicates the maximum pseudorapidity of secondary hadrons from photon fragmentation and is related to the fraction $x_{p}$ of the initial proton momentum carried by the diffractively scattered proton via $\eta_{\max }^{*} \sim \ln \left(1-x_{p}\right)+C$ [36]. The comparison of the $\eta_{\max }^{*}$ distributions for DIS and photoproduction interactions is shown in Fig. đtb. The figure shows spectra which fall off rapidly from $\eta_{\text {max }}^{*}=1$ with decreasing $\eta_{\text {max }}^{*}$. This part of the spectra can be described by non-diffractive DIS and photoproduction interactions as demonstrated in [3, 37]. For $\eta_{\max }^{*} \sim-1$ the spectra level off to a constant plateau. The events with this and lower $\eta_{\max }^{*}$ values have been shown to originate predominantly from a diffractive mechanism [38, 37]. Below $\eta_{\max }^{*}<-3$ the photoproduction and DIS data are affected differently by the detector acceptance and therefore not included in this analysis. As a consequence of this cut the low mass resonance region is avoided as well. For diffractive events the distribution $1 / N \cdot d N / d \eta_{\max }^{*} \sim\left(1 / \sigma_{t o t}\right)\left(d \sigma / d\left(1-x_{p}\right)\right)\left(1-x_{p}\right)$ is expected to be roughly independent of $\eta_{\max }^{*}$ if the diffractive cross section $d \sigma / d\left(1-x_{p}\right)$ is approximately inversely proportional to $\left(1-x_{p}\right)$, see ref. [38] 3. This lack of dependence of $1 / N \cdot d N / d \eta_{\max }^{*}$ on $\eta_{\max }^{*}$ is itself often taken as the signature of diffraction [39].

Fig. $4 \mathrm{~b}$ shows clearly that indeed at low $\eta_{\max }^{*}$ both for DIS and photoproduction the differential distribution $1 / N \cdot d N / d \eta_{\max }^{*}$ is roughly independent of $\eta_{\text {max }}^{*}$. Furthermore the relative contribution of the photon high mass diffractive dissociation for photoproduction and DIS interactions is found to be the same to within 15-20\%. This agreement is not affected by detector effects since for a given $\eta_{\text {max }}^{*}$ value largely the same detector regions are explored in DIS and photoproduction interactions. This observation is at the same level of agreement as measurements made for hadron-hadron interactions, and is in accord with the expectation of approximate factorization of high mass diffractive cross sections, as observed in hadron collisions and explained with triple Regge phenomenology [15]: the ratio of the differential diffractive cross section, $d \sigma / d\left(1-x_{p}\right)$, to the total cross section is approximately independent of the type of dissociating hadron. The fact that this factorization rule also seems to hold for virtual photon interactions gives an additional argument in favour of the validity of a universal hadron-like description of low- $x$ DIS, real photoproduction, and hadron-hadron collisions.

\section{Conclusions}

The comparison of photoproduction and low- $x$ DIS data at the ep collider HERA reveals striking similarities in the energy flow of the hadronic final state and relative rate of high

\footnotetext{
${ }^{3}$ It was shown that for DIS events $d \sigma / d\left(1-x_{p}\right)$ is proportional to $\left(1-x_{p}\right)^{n}$ with $n=1.19 \pm 0.06 \pm 0.07$
} 
mass diffractive dissociation of the photon. The $W$ dependence of the transverse energy density in the central rapidity region is found to be similar to that seen in high energy hadron-hadron collisions. These findings are consistent with the hadronic picture of the photon which can therefore be considered to be complementary to the conventional deepinelastic scattering picture, even at large $Q^{2}$ values. This picture gives a description of the transition region from the high $Q^{2}$ perturbative region to the low $Q^{2}$ non-perturbative region, and provides a basis for a universal description of hadron-hadron, real photonhadron and virtual photon-hadron high energy interactions.

\section{Acknowledgements}

We are grateful to the HERA machine group whose outstanding efforts made this experiment possible. We appreciate the immense effort of the engineers and technicians who constructed and maintained the detector. We thank the funding agencies for their financial support of the experiment. We wish to thank the DESY directorate for the support and hospitality extended to the non-DESY members of the collaboration. We thank further J. Bartels, A. Kaidalov, J. Kwiecinski and M. Ryskin for useful discussions.

\section{References}

[1] J.J. Sakurai, Phys. Rev. Lett. 22 (1969) 981.

[2] H1 Collab., I. Abt et al., Phys. Lett. B328 (1994) 176.

[3] H1 Collab., T. Ahmed et al., Nucl. Phys. B429 (1994) 477.

[4] ZEUS Collab., M. Derrick et al., Phys. Lett. B315 (1993) 481.

[5] NMC Collab., P. Amaudruz et al., Z. Phys. C51 (1991) 387;

E665 Collab., M.R. Adams et al., Phys. Rev. Lett. 68 (1992) 3266.

[6] T.H. Bauer et al., Rev. Mod. Phys. 50 (1978) 261.

[7] B.L. Ioffe, Phys. Lett. B30 (1969) 123.

[8] V. Del Duca, S.J. Brodsky and P. Hoyer, Phys. Rev. D46 (1992) 931.

[9] N. N. Nikolaev, B.G. Zakharov, Phys. Lett. B327 (1994) 149.

[10] J.D. Bjorken and J.B. Kogut, Phys. Rev. D8 (1973) 1341.

[11] J. Kwiecinski and B. Badelek, Z. Phys. C43 (1989) 251.

[12] H1 Collab., T. Ahmed et al., Nucl. Phys. B439 (1995) 471.

[13] J.D.Bjorken, SLAC Summer school SLAC-167 (1973) 1.

[14] NA22 Collab., M. Adamus et al., Z. Phys C39 (1988) 311.

[15] K. Goulianos, Phys. Rep. 101 (1983) 169.

[16] K. Goulianos, Nucl. Phys. B (Proc. Suppl.) 12 (1990) 110.

[17] H1 Collab., I. Abt et al., DESY preprint 93-103 (1993), to be published in Nucl. Instr. and Meth.

[18] H1 Calorimeter Group, B. Andrieu et al., Nucl. Instr. and Meth. A336 (1993) 460.

[19] H1 Calorimeter Group, B. Andrieu et al., Nucl. Instr. and Meth. A350 (1994) 57. 
[20] H1 Calorimeter Group, B. Andrieu et al., Nucl. Instr. and Meth. A336 (1993) 499.

[21] H1 BEMC group, "The H1 backward calorimeter BEMC", to be submitted to Nucl. Instr. and Meth.

[22] H1 Collab., I. Abt et al., Nucl. Phys. B407 (1993) 515.

[23] H1 Collab., T. Ahmed et al., Phys. Lett. B346 (1995) 415.

[24] H1 Collab., T. Ahmed et al., Nucl. Phys. B445 (1995) 195.

[25] R. Engel, Proceedings of the XXIXth Recontre de Moriond (1994) 321.

[26] L. Lönnblad, Computer Phys. Comm. 71 (1992) 15.

[27] P. Aurenche et al., Phys.Rev. D45 (1992) 92.

[28] G. Gustafson, Ulf Petterson, Nucl. Phys. B306 (1988) 746;

G. Gustafson, Phys. Lett. B175 (1986) 453;

B. Andersson et al., Z.Phys. C43 (1989) 625.

[29] G. Ingelman, Proc. HERA workshop, Eds. W. Buchmüller and G. Ingelman, Hamburg (1991) Vol. 3,1366 .

[30] H1 Collab., S. Aid et al., DESY preprint 95-086 (1995).

[31] L. Lönnblad, Ariadne Version 4.06, Program Manual.

[32] H1 Collab., S. Aid et al., DESY preprint 95-108 (1995), submitted to Phys. Lett.

[33] S. Brodsky et al., Phys Rev. D50 (1994) 3134.

[34] UA1 Collab., C. Albajar et al., Nucl.Phys. B335, (1990) 261.

[35] A. De Roeck, Inclusive Particle Production in Hadron-Proton Interactions at $250 \mathrm{GeV} / \mathrm{c}$, Phd. Thesis, Antwerpen (1988).

[36] A.B.Kaidalov, Phys.Rep. 50 (1979) 157.

[37] H1 Collab., T. Ahmed et al., Nucl. Phys. B435 (1995) 3.

[38] H1 Collab., T. Ahmed et al., Phys. Lett. B348 (1995) 681.

[39] J.D. Bjorken, Proceedings of the International Workshop on Deep Inelastic Scattering and Related Subjects, Eilat, editor A. Levy, World Scientific (1994) 151, and SLAC preprint SLAC-PUB-6477 (1994). 